\title{
DISTRIBUTION TRANSFORMERS MODELING WITH ANGULAR DISPLACEMENT - ACTUAL VALUES AND PER UNIT ANALYSIS
}

\author{
Dario E. Rodas R.* \\ drodaseutp.edu.co \\ Luis F. Ochoa ${ }^{\ddagger}$ \\ luis_ochoadieee.org \\ *Universidad Tecnológica de Pereira (UTP) - Pereira, Colombia \\ Phone: +57 63212044 \\ $\dagger$ UNESP - Campus de Ilha Solteira \\ Av. Brasil 56, Ilha Solteira - SP, Brazil \\ Phone: +551837431169 \\ ${ }^{\ddagger}$ University of Edinburgh \\ Edinburgh EH9 3JL, UK \\ Phone: +44 1316505575
}

Antonio Padilha-Feltrin ${ }^{\dagger}$

padilhaddee.feis.unesp.br

\begin{abstract}
In this work a detailed modeling of three-phase distribution transformers aimed at complementing well-known approaches is presented. Thus, incidence of angular displacement and tapping is taken into account in the proposed models, considering both actual values and per unit. The analysis is based on minimal data requirement: solely short-circuit admittance is needed since three-phase transformers are treated as non-magnetically-coupled single-phase transformers. In order to support the proposed methodology, results obtained through laboratory tests are presented.
\end{abstract}

KEYWORDS: Transformer modeling, angular displacement, distribution systems.

Artigo submetido em 04/05/2006

1a. Revisão em 22/08/2006

2a. Revisão em 15/05/2007

Aceito sob recomendação do Editor Associado

Prof. Denis Vinicius Coury

\section{NOMENCLATURE}

$V_{x}$ : Voltage corresponding to winding $x, x \in[1,6]$.

$V_{a}, V_{b}, V_{c}$ : Voltages corresponding to primary-side phases $a, b$ and $c$.

$V_{A}, V_{B}, V_{C}$ : Voltages corresponding to secondary-side phases $A, B$ and $C$.

$Z_{1}, Z_{2}$ : Impedances corresponding to windings 1 and 2 , respectively.

$Z_{m}$ : Mutual impedance between windings 1 and 2.

$I_{1}, I_{2}$ : Currents corresponding to windings 1 and 2 , respectively.

$n_{1}, n_{2}$ : Turns corresponding to windings 1 and 2, respectively.

$Y t$ : Short-circuit admittance referred to the primary side.

$a$ : Transformation ratio.

$[Y p]$ : Primitive admittance matrix. 
$S_{n}$ : Base power.

$V_{n_{1}}, V_{n_{2}}$ : Base voltages corresponding to windings 1 and 2, respectively.

$Z_{\text {base }}:$ Base impedance.

$R, X$ : Resistance and reactance, respectively.

$\alpha, \beta$ : Tap variations at the primary and secondary sides respect to their nominal values, respectively.

[Ybus]: Admittance matrix.

$\left[Y_{p p}\right],\left[Y_{p s}\right],\left[Y_{s p}\right],\left[Y_{s s}\right]:$ Submatrices of matrix $[Y b u s]$.

$[N]$ : Connection matrix.

$\left[N_{p p}\right],\left[N_{s s}\right]$ : Submatrices of matrix $[N]$.

$g$ : Grounded.

\section{INTRODUCTION}

It is well known that transformers are fundamental elements of power systems, requiring proper models so accurate analyses of the network can be performed. In transmission system, transformer modeling is single-phase based since its operation is considered to be balanced. On the other hand, given the inherent unbalance nature of distribution systems, a detailed modeling of distribution transformers requires a three-phase approach taking into account $a-b-c$ components, including neutral and ground effects. Thus, three-phase transformer modeling has been the focus of several researches (Chen et alli, 1991; Chen and Chang, 1992; Chen et alli, 1996; Chen and Chang, 1996; Baran and Staton, 1997; Kersting et alli, 1999; Kersting, 2002; Dugan, 2003; Wang et alli, 2004; Xiao et alli, 2006). Nonetheless, models found in the literature still lack of details regarding some connections, angular displacement and tapping.

Depending on the power flow algoithm adopted for the distribution network analysis, transformer models may lead to convergence problems. Consequently, some proposals are given in the literature in order to minimize this effect (Chen and Chang, 1996; Baran and Staton, 1997; Kersting et alli, 1999; Wang et alli, 2004; Xiao et alli, 2006). Evaluation of the impacts that certain transformer models present on the power flow analysis is out of the scope of this paper.

Although transformer models presented in 1991 by Chen et alli have been widely used by researchers and commercial software, their applicability outside the US is limited since the angular displacement is commonly restricted to two clock hours: 1 and 11, depending on whether it is a step-up or stepdown arrangement.
The objective of this paper is to improve and to make as general as possible widely-accepted transformer models, considering angular displacement as well as tapping in both primary and secondary windings. It is also demonstrated that distribution transformers can be modeled as power transformers when analyzing variable taps. Proposed three-phase transformer models allow including angular displacement, which is a basic tool for generalizing transformer models to other different clock hours or even vector groups. A discussion about circuit and matrix modeling considering the variables in actual values and in per unit is also included. Models in actual values (Siemens) are fully described since they allow obtaining directly all major parameters involved in the transformer modeling, leading to a better understanding of the per unit analysis. Moreover, in order to validate the proposed methodology, results from laboratory tests are presented.

Generally speaking, there two ways to model distribution transformer. One requires a large quantity of data (Gorman and Grainger, 1992), which is often difficult to obtain. The other way considers the use of minimal information (only the short-circuit admittance is needed) whereas the core is modeled as a load (Dugan, 2003; Chen et alli, 1991). The modeling presented in this work is classified in the latter group. Moreover, this proposal allows the modeling of all kinds of winding connections given by the American National Standards Institute (ANSI) and the International Electrotechnical Commission (IEC).

The structure of this paper is as follows: section 3 presents the fundamental concepts for single-phase transformers, whereas in section 4 the incidence of taps on single-phase transformers is addressed. Distribution transformer analysis is presented in section 5. Finally, in section 6 transformer bank modeling is addressed taking into account actual values and per unit, as well as angular displacement and tapping incidence.

\section{SINGLE-PHASE TRANSFORMER FUNDAMENTAL CONCEPTS}

A single-phase transformer model based on matrices and, initially, considering its nominal values (winding 1 with $n_{1}$ turns and winding 2 with $n_{2}$ turns), as presented in figure 1, is given by:

$$
\left[\begin{array}{l}
V_{1} \\
V_{2}
\end{array}\right]=\left[\begin{array}{cc}
Z_{1} & Z_{m} \\
Z_{m} & Z_{2}
\end{array}\right] \cdot\left[\begin{array}{c}
I_{1} \\
I_{2}
\end{array}\right]
$$

These values are winding based, i.e. primitive impedance is used. 


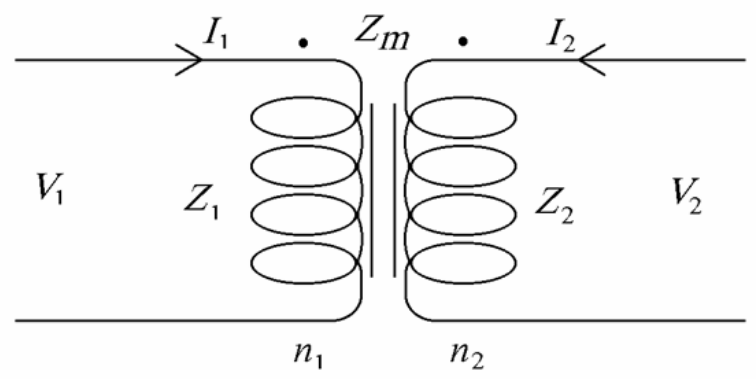

Figure 1: Single-phase transformer without tapping.

$$
Y t=\frac{Z_{2}}{Z_{1} Z_{2}-Z_{m}^{2}}
$$

Equations (1) and (2) could be either based on actual values or p.u. Regarding the case of actual values in equation (2), $Y t$ is the short-circuit admittance referred to the primary. Then, the primitive admittance matrix is:

$$
[Y p]=\left[\begin{array}{cc}
Y t & -a Y t \\
-a Y t & a^{2} Y t
\end{array}\right]
$$

where $a=\frac{n_{1}}{n_{2}}$.

Matrix $[Y p]$ in p.u. is obtained by dividing each term of equation (3), whose units are Siemens, by its corresponding base values:

$Y_{p-b a s e}=\frac{S_{n}}{V_{n_{1}}^{2}}, \quad Y_{p s-b a s e}=\frac{S_{n}}{V_{n_{1}} V_{n_{2}}}$ and $Y_{s-b a s e}=\frac{S_{n}}{V_{n_{2}}^{2}}$.

where $p$ : primary and $s$ : secondary.

Then, substituting $a=\frac{V_{n_{1}}}{V_{n_{2}}}$, the primitive admittance $[Y p]$ in p.u. is obtained:

$$
[Y p]_{p u}=\left[\begin{array}{cc}
y_{p u} & -y_{p u} \\
-y_{p u} & y_{p u}
\end{array}\right]
$$

\section{GENERAL MODEL FOR A SINGLE-PHASE TRANSFORMER WITH VARIABLE TAPS}

When taps vary, fundamentally two parameters experience alterations: a) The voltage at the secondary side of the transformer due to the new transformation ratio, and;

b) The transformer short-circuit impedance since the inductive reactance is a function of the squared number of turns. When modifying the number of turns, the resistance is also affected.

When nominal values are considered (taps at their nominal positions in both primary and secondary sides), short-circuit tests are performed in order to obtain the transformer's nominal admittance: $Y t_{\text {nom }}$.

\subsection{Off-nominal Taps incidence on Transformer's Impedance}

In power transformers, where the resistance can be neglected $(Z \approx X)$, tap variations modify the impedances according to the new number of turns and quadratically since the inductance is a function of the squared number of turns.

Let $\alpha$ and $\beta$ be the tap variations at the primary and secondary sides respect to their nominal values, respectively. Thus, when taps are altered, the new transformer ratio is:

$$
a_{\text {new }}=a \cdot \frac{\alpha}{\beta} .
$$

Consequently, $Z_{1-\text { new }}=\alpha^{2} Z_{1-\text { nom }}, Z_{2-\text { new }}=$ $\beta^{2} Z_{2-n o m}$ and $Z_{m-n e w}=\alpha \beta Z_{m-n o m}$.

This is valid for both actual values and p.u. as long as the nominal base values of $Z_{\text {base }}$ are kept constant. The transformer's new admittance will be:

$$
Y t_{\text {new }}=\frac{Z_{2-\text { new }}}{Z_{1-\text { new }} Z_{2-\text { new }}-Z_{m-\text { new }}^{2}}
$$

$$
Y t_{\text {new }}=\frac{\beta^{2} Z_{2-\text { nom }}}{\alpha^{2} Z_{1-\text { nom }} \cdot \beta^{2} Z_{2-\text { nom }}-\alpha^{2} \beta^{2} Z_{m-\text { nom }}^{2}}
$$

$$
Y t_{\text {new }}=\frac{Z_{2-\text { nom }}}{\alpha^{2}\left(Z_{1-\text { nom }} Z_{2-\text { nom }}-Z_{m-\text { nom }}^{2}\right)}
$$

Then, using equation (2) and considering nominal values:

$$
Y t_{\text {new }}=\frac{Y t_{\text {nom }}}{\alpha^{2}}
$$




\subsection{Off-nominal Taps incidence on Transformer's Primitive Admittance}

In order to avoid mistakes in the analysis, the process performed in section 3 is repeated taking into account a new number of turns for both primary and secondary sides. Thus,

$$
\begin{aligned}
& n_{1}^{\prime}=\alpha \cdot n_{1} \\
& n_{2}^{\prime}=\beta \cdot n_{2}
\end{aligned}
$$

When the short-circuit test is carried out, the voltage in the secondary side is null $\left(V_{2}^{\prime}=0\right)$. Then, according to equation (5), the transformer's short-circuit admittance is:

$$
Y t^{\prime}=\frac{Z_{2}^{\prime}}{Z_{1}^{\prime} Z_{2}^{\prime}-Z_{m}^{\prime 2}}=\frac{Y t}{\alpha^{2}}
$$

In addition, the following expressions are also obtained:

$$
\begin{gathered}
\frac{Z_{1}^{\prime}}{Z_{m}^{\prime}}=\frac{n_{1}^{\prime}}{n_{2}^{\prime}}=\frac{\alpha \cdot n_{1}}{\beta \cdot n_{2}}=a \frac{\alpha}{\beta} \\
\frac{Z_{m}^{\prime}}{Z_{2}^{\prime}}=\frac{n_{1}^{\prime}}{n_{2}^{\prime}}=\frac{\alpha \cdot n_{1}}{\beta \cdot n_{2}}=a \frac{\alpha}{\beta} \\
\frac{Z_{1}^{\prime}}{Z_{2}^{\prime}}=\left(\frac{n_{1}^{\prime}}{n_{2}^{\prime}}\right)^{2}=\left(\frac{\alpha \cdot n_{1}}{\beta \cdot n_{2}}\right)^{2}=a^{2}\left(\frac{\alpha}{\beta}\right)^{2}
\end{gathered}
$$

Finally, it is obtained:

$$
[Y p]^{\prime}=\left[\begin{array}{cc}
Y t^{\prime} & -Y t^{\prime} \cdot a \cdot \frac{\alpha}{\beta} \\
-Y t^{\prime} \cdot a \cdot \frac{\alpha}{\beta} & Y t^{\prime} \cdot a^{2} \cdot\left(\frac{\alpha}{\beta}\right)^{2}
\end{array}\right]
$$

Then, substituting $Y t^{\prime}$ according to equation (5):

$$
[Y p]_{\text {new }}=\left[\begin{array}{cc}
\frac{Y t_{\text {nom }}}{\alpha^{2}} & -\frac{a Y t_{\text {nom }}}{\alpha \beta} \\
-\frac{a Y t_{\text {nom }}}{\alpha \beta} & \frac{a^{2} Y t_{\text {nom }}}{\beta^{2}}
\end{array}\right]
$$

Equation (13) is in Siemens and $Y t_{n o m}$ stands for the nominal short-circuit admittance referred to the primary side. For analysis in p.u., the following expression should be used:
Table 1: Values obtained in the tests

\begin{tabular}{|c|c|c|c|c|c|c|}
\hline Tap & $\alpha$ & $\begin{array}{c}\text { Current } \\
(\mathrm{A})\end{array}$ & $\begin{array}{c}\text { Voltage } \\
(\mathrm{V})\end{array}$ & $\begin{array}{c}\text { Power } \\
(\mathrm{W})\end{array}$ & $\mathrm{R}(\Omega)$ & $\mathrm{X}(\Omega)$ \\
\hline 1 & 1.025 & 0.790 & 243 & 66 & 105.75 & 288.844 \\
\hline 2 & 1.000 & 0.830 & 242 & 70 & 101.61 & 273.287 \\
\hline 3 & 0.975 & 0.874 & 242 & 74 & 97.77 & 259.388 \\
\hline 4 & 0.950 & 0.974 & 242 & 85 & 90.34 & 231.742 \\
\hline
\end{tabular}

$$
[Y p]_{p u-n e w}=\left[\begin{array}{cc}
\frac{Y t_{p u-n o m}}{\alpha^{2}} & -\frac{Y t_{p u-n o m}}{\alpha \beta} \\
-\frac{Y t_{p u-n o m}}{\alpha \beta} & \frac{Y t_{p u-n o m}}{\beta^{2}}
\end{array}\right]
$$

\section{DISTRIBUTION TRANSFORMERS}

In this section a real-life distribution transformer (20 KVA, $13200 \mathrm{~V} / 240 \mathrm{~V}$ ) is analyzed by means of laboratory tests. Taps at the primary side are:

Tap1 = 13530 V (2.5\%);

Tap2 = $13200 \mathrm{~V}$ (nominal);

Tap3 = 12870 V $(-2.5 \%)$;

Tap4 = $12540 \mathrm{~V}(-5.0 \%)$;

Tap5 $=12210 \mathrm{~V}(-7.5 \%)$.

The following analysis will consider solely variations at the primary side, which is the case of distribution network transformers. Consequently, $\beta=1$. These short-circuit tests were carried out considering the transformer's nominal current.

\subsection{Laboratory Tests}

Tests carried out varying the available taps of the transformer led to the results shown in table 1.

Considering the impedance $\left(Z_{t}=R_{t}+j X_{t}\right)$ of the most distant tap from the nominal position (Tap 5, $\alpha=0.925$ ), one can compute the corresponding short-circuit admittance referred to the primary side. Thus,

$Z_{t}^{\prime}=90.34+j 231.742 \Omega$, then

$$
Y_{t}^{\prime}=\left(Z_{t}^{\prime}\right)^{-1}=1.46 \cdot 10^{-3}-j 3.746 \cdot 10^{-3} \text { Siemens. }
$$

Taking into account that in this case $a=13200 / 240=$ 
55 and using equation (12), the transformer's primitive admittance matrix is obtained:

$$
[Y p]=\left[\begin{array}{cc}
4.02 \cdot 10^{-3} \angle-68.702^{\circ} & -0.2045 \angle-68.702^{\circ} \\
-0.2045 \angle-68.702^{\circ} & 10.406 \angle-68.702^{\circ}
\end{array}\right]
$$

Siemens.

In order to verify the performance of the power transformer model applied on distribution transformers, equation (13) is used to compute the primitive admittance matrix for Tap 5 (table 1). The nominal short-circuit admittance is calculated as follows:

$Z t_{\text {nom }}=101.61+j 273.287 \Omega$, then

$Y t_{\text {nom }}=\left(Z t_{\text {nom }}\right)^{-1}=3.4298 \cdot 10^{-3} \angle-69.6^{\circ}$ Siemens.

Hence, considering also $a=55$ and $\alpha=0.925$, the following matrix is calculated:

$$
[Y p]=\left[\begin{array}{cc}
4.008 \cdot 10^{-3} \angle-69.6^{\circ} & -0.2039 \angle-69.6^{\circ} \\
-0.2039 \angle-69.6^{\circ} & 10.375 \angle-69.6^{\circ}
\end{array}\right]
$$

The comparison of both results prove the good performance of the method based on power transformers since the errors in magnitude do not exceed $0.3 \%$, whereas for the angle this value is $1.3 \%$. Consequently, given the accuracy obtained with a transformer of relatively low capacity (20 KVA), it is expected that most distribution transformers (commonly with capacities higher than 20KVA) present better results. Offnominal load cenarios are also expected to exhibit similar performances.

\subsection{Modeling with Taps at the Primary Side}

When taps are located at the primary side, the winding at the secondary side (winding 2) is not modified. Therefore, the resistance referred to the secondary side $\left(R_{2}\right)$ is not modified either. Nevertheless, when taps vary, $R_{2}$ referred to the primary side should take into account the transformation ratio $a \alpha$. In addition, considering that the resistance of the primary side varies according to the number of turns, in other words, the resistance varies linearly with $\alpha$ and $R_{2}^{\prime} \approx R_{1}$, the new resistance is obtained as follows:

$$
R_{t-n e w}=\alpha R_{1}+(a \alpha)^{2} R_{2}=\alpha R_{1}+\left(a^{2} R_{2}\right) \alpha^{2}
$$

$$
\begin{gathered}
R_{t-\text { new }}=\alpha R_{1}+\left(R_{2}^{\prime}\right) \alpha^{2} \cong \alpha R_{1}+\left(R_{1}\right) \alpha^{2} \\
R_{t-\text { new }}=R_{1}\left(\alpha+\alpha^{2}\right)
\end{gathered}
$$

$$
\begin{gathered}
\frac{R_{t-\text { new }}}{R_{t-\text { nom }}}=\frac{R_{1}\left(\alpha+\alpha^{2}\right)}{2 R_{1}}=\frac{\left(\alpha+\alpha^{2}\right)}{2} \\
R_{t-\text { new }}=R_{t-\text { nom }} \cdot\left(\alpha+\alpha^{2}\right) / 2
\end{gathered}
$$

On the other hand, the reactance varies quadratically:

$$
X_{t-n e w}=\alpha^{2} X_{1}+(a \alpha)^{2} X_{2}=\alpha^{2} X_{1}+\left(a^{2} X_{2}\right) \alpha^{2}
$$

$$
X_{t-n e w}=\alpha^{2} X_{1}+\left(X_{2}^{\prime}\right) \alpha^{2} \cong \alpha^{2} X_{1}+\left(X_{1}\right) \alpha^{2}
$$

$$
X_{t-n e w}=X_{1}\left(2 \alpha^{2}\right)
$$

$$
\begin{gathered}
\frac{X_{t-\text { new }}}{X_{t-\text { nom }}}=\frac{X_{1}\left(2 \alpha^{2}\right)}{2 X_{1}}=\alpha^{2} \\
X_{t-\text { new }}=X_{t-\text { nom }} \cdot \alpha^{2}
\end{gathered}
$$

Thus, the distribution transformer model for any $\alpha$ is:

$$
Z_{t}=R_{t-n o m}\left(\alpha+\alpha^{2}\right) / 2+j X_{t-n o m} \alpha^{2}
$$

Another way to approach the transformer modeling with taps at the primary side is that when distribution transformers are considered as power transformers. In this case, selfquadratic variation of the reactance $\left(\alpha^{2}\right)$ is applied to the whole impedance, i.e. it is applied on the resistance as well.

In order to compare both approaches presented above, and given that the resistance is the parameter being considered differently, the multiplying factors of $R_{t-n o m}$ are presented in table 2: 
Table 2: Multiplying factors of $R_{t-n o m}$.

\begin{tabular}{|c|c|c|}
\hline$\alpha$ & $\left(\alpha+\alpha^{2}\right) / 2$ & $\alpha^{2}$ \\
\hline 0.925 & 0.89 & 0.8556 \\
\hline 1.025 & 1.038 & 1.0506 \\
\hline
\end{tabular}

Table 3: Measured impedance vs. estimated impedance.

\begin{tabular}{|c|c|c|c|c|c|}
\hline \multirow{2}{*}{$\alpha$} & \multicolumn{2}{|c|}{ measured $(\Omega)$} & \multicolumn{3}{|c|}{ estimated $(\Omega)$} \\
\cline { 2 - 6 } & $\mathrm{R}$ & $\mathrm{X}$ & $R\left[\left(\alpha+\alpha^{2}\right) / 2\right]$ & $R\left[\alpha^{2}\right]$ & $\mathrm{X}$ \\
\hline 1.025 & 105.75 & 288.844 & 105.45 & 106.75 & 287.12 \\
\hline 0.975 & 97.77 & 259.388 & 97.83 & 96.59 & 259.80 \\
\hline 0.950 & 94.52 & 245.475 & 94.12 & 91.70 & 246.64 \\
\hline 0.925 & 90.34 & 231.742 & 90.46 & 86.94 & 233.83 \\
\hline
\end{tabular}

Since results are similar, approximations can be used for the resistance. Moreover, generally speaking, the whole impedance can be approximated. Thus, $Y_{\text {new }}=Y_{\text {nom }} / \alpha^{2}$.

Results obtained through laboratory measurements (table 1) and through equations (15), (16), and the approximation above presented, are shown in table 3 .

Strictly speaking, the reactive part of the model adjusts properly the estimated values compared to those measured in laboratory. It is also observed that, regarding the resistive part, the first approach, equation (15), follows more accurately (almost perfect adjustment) the measured values. Nevertheless, due to systematic problems when forming matrix [Ybus], it is recommendable to consider the distribution transformer as power transformer $\left(\alpha^{2}\right)$ since embedded errors are relatively small.

\section{TRANSFORMER BANKS MODELING}

This section presents in a general manner the mathematical models of three-phase transformers obtained from using single-phase transformers as a three-phase bank. Incidence of the transformer's angular displacement on its corresponding model is also addressed.

Magnetization impedance of a transformer is quite large and is neglected for voltage regulation studies. This approximation does not affect power flow results. Iron losses obtained through the open-circuit test can be modeled by adding them to the actual load.

Modeling a three-phase transformer considering all magnetic couplings may require data from several short-circuit tests. Therefore, it is a common procedure to consider three-phase distribution transformers as a bank of three single-phase transformers, magnetically isolated from each other and connected properly to achieve the connection modeling under analysis (Kersting, 2002). Thus, required information for modeling the three-phase transformer is reduced exclusively to the short-circuit test for solely one transformer, information that is always available.

In general, it is recommended to obtain matrix $[Y p]$ directly from the short-circuit tests since, otherwise, in order to avoid numerical instability problems it would be necessary to obtain extremely accurate measurements of the $Z$ parameters.

The following subsections 6.1 and 6.2 are aimed at presenting the procedures needed to obtain matrix [Ybus] for both approaches, Siemens and per unit. Two application examples will be presented thereafter. Subsections 6.4 and 6.5 present the connections summary for various types of connections and the transformer modeling considering the ideal ordering for the primitive admittance and connection matrices, respectively. The latter is important since conclusions will be drawn in order to model the incidence of the angular displacement, presented in subsection 6.6. Finally, the incidence of taps in the three-phase transformer modeling is addressed in subsection 6.7.

\subsection{Approach in Siemens}

To obtain matrix $[$ Ybus $]$ in actual values the following steps should be performed:

1. A 6 by 6 primitive admittance matrix $[Y p]$ is formed in Siemens, composed by three decoupled singlephase transformers. First, third and fifth rows and columns represent the primary sides of transformers, whereas the remaining rows and columns represent the corresponding secondary sides.

$$
\begin{aligned}
& {[Y p]=} \\
& =\left[\begin{array}{cccccc}
Y_{t} & -a Y_{t} & 0 & 0 & 0 & 0 \\
-a Y_{t} & a^{2} Y_{t} & 0 & 0 & 0 & 0 \\
0 & 0 & Y_{t} & -a Y_{t} & 0 & 0 \\
0 & 0 & -a Y_{t} & a^{2} Y_{t} & 0 & 0 \\
0 & 0 & 0 & 0 & Y_{t} & -a Y_{t} \\
0 & 0 & 0 & 0 & -a Y_{t} & a^{2} Y_{t}
\end{array}\right]
\end{aligned}
$$

This step is general for all possible connections in Siemens and, meanwhile, considering nominal taps. At this stage the three-phase transformer is not yet connected. 
2. For a particular connection under study the corresponding connection matrix $[N]$, which relates windings voltages to nodal voltages and considers the angular displacement, should be selected.

3. The following formula is applied:

$$
[\text { Ybus }]=[N]^{t} \cdot[Y p] \cdot[N]
$$

\subsection{Approach in Per Unit}

Matrix $[$ Ybus $]$ can be also computed in per unit following a similar procedure to that presented for actual values:

1. Same as step 1 of previous subsection, but considering matrix $[Y p]$ in p.u., using three submatrices as in equation (4).

2. Same as step 2 of previous subsection.

3. Same as step 3 of previous subsection.

4. Matrix $[Y b u s]$ can be presented in terms of its submatrices:

$$
[Y \text { bus }]=\left[\begin{array}{ll}
{\left[Y_{p p}\right]} & {\left[Y_{p s}\right]} \\
{\left[Y_{s p}\right]} & {\left[Y_{s s}\right]}
\end{array}\right]
$$

where $\left[Y_{p p}\right]$ stands for the primary side, $\left[Y_{s s}\right]$ stands for the secondary side and $\left[Y_{p s}\right]$ and $\left[Y_{s p}\right]$ for the primarysecondary and secondary-primary, respectively. These submatrices should be corrected due to changes in base voltages according to the corresponding connection, as follows:

Wye-wye: [Ybus] remains without alteration.

Wye-delta: Base voltage at the secondary changes, consequently $[Y$ bus $]$ in p.u. should be adapted in terms of its submatrices. Thus, $\left[Y_{p p}\right]$ remains the same, $\left[Y_{p s}\right]$ and $\left[Y_{s p}\right]$ are divided by $\sqrt{3}$, and $\left[Y_{s s}\right]$ is divided by 3 .

Delta-delta: Both base voltages at the primary and secondary sides change, therefore the whole matrix [Ybus] should be divided by 3 .

Delta-wye: Base voltage at the primary side changes. Thus, $\left[Y_{p p}\right]$ is divided by $3,\left[Y_{p s}\right]$ and $\left[Y_{s p}\right]$ are divided by $\sqrt{3}$, and $\left[Y_{s s}\right]$ remains the same.

\subsection{Examples}

In this subsection two distribution transformers, with grounded and isolated neutral, will be analyzed aimed at illustrating the calculation details of matrix [Ybus] considering both approaches, actual values and per unit.

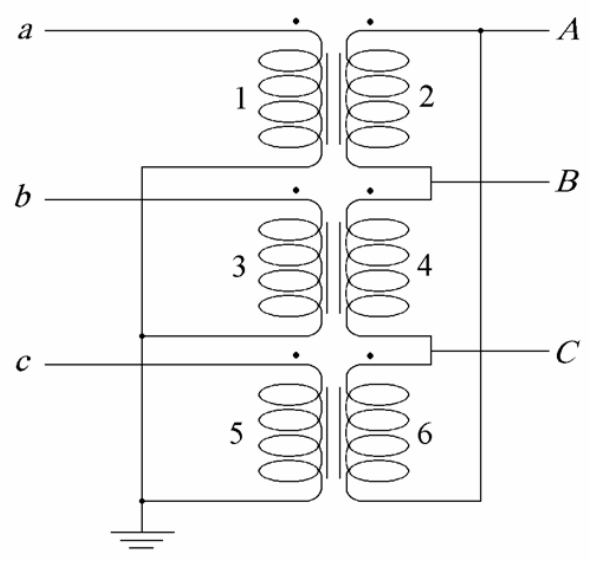

Figure 2: Grounded-neutral Yd1 Transformer.

\subsubsection{Grounded-neutral Yd1 Transformer}

The electrical scheme of the wye-delta transformer with angular displacement of $30^{\circ}$ ( $1 \mathrm{o}$ 'clock) is presented in figure 2.

Firstly, winding voltages are presented as a function of node voltages. Numbers are referred to winding voltages $(1,3$ and 5 for the primary side and 2, 4 and 6 for the secondary side) and letters $(a, b, c, A, B, C)$ are referred to nodal voltages respect to the reference (ground), as follows:

$$
\left[\begin{array}{l}
V_{1} \\
V_{2} \\
V_{3} \\
V_{4} \\
V_{5} \\
V_{6}
\end{array}\right]=\left[\begin{array}{cccccc}
1 & 0 & 0 & 0 & 0 & 0 \\
0 & 0 & 0 & 1 & -1 & 0 \\
0 & 1 & 0 & 0 & 0 & 0 \\
0 & 0 & 0 & 0 & 1 & -1 \\
0 & 0 & 1 & 0 & 0 & 0 \\
0 & 0 & 0 & -1 & 0 & 1
\end{array}\right] \cdot\left[\begin{array}{c}
V_{a} \\
V_{b} \\
V_{c} \\
V_{A} \\
V_{B} \\
V_{C}
\end{array}\right]
$$

The matrix which is multiplying the nodal voltages is called connection matrix $[N]$. Then, solving equation (19) for this particular connection, the admittance matrix for those six nodes is expressed by:

$$
\begin{gathered}
{[\text { Ybus }]=} \\
=\left[\begin{array}{cccccc}
Y_{t} & 0 & 0 & -a Y_{t} & a Y_{t} & 0 \\
0 & Y_{t} & 0 & 0 & -a Y_{t} & a Y_{t} \\
0 & 0 & Y_{t} & a Y_{t} & 0 & -a Y_{t} \\
-a Y_{t} & 0 & a Y_{t} & 2 a^{2} Y_{t} & -a^{2} Y_{t} & -a^{2} Y_{t} \\
a Y_{t} & -a Y_{t} & 0 & -a^{2} Y_{t} & 2 a^{2} Y_{t} & -a^{2} Y_{t} \\
0 & a Y_{t} & -a Y_{t} & -a^{2} Y_{t} & -a^{2} Y_{t} & 2 a^{2} Y_{t}
\end{array}\right]
\end{gathered}
$$


At this point, the problem has been solved in Siemens, considering $a$ equal to the nominal winding ratio and $Y_{t}$ as the short-circuit admittance (in Siemens) referred to the primary side.

If values in p.u. are required, firstly, equation (19) should consider $a=1$ and $Y_{t}$ in p.u. Secondly, due to the wye-delta connection, submatrices $\left[Y_{p s}\right]$ and $\left[Y_{s p}\right]$ are divided by $\sqrt{3}$, and $\left[Y_{s s}\right]$ is divided by 3 . Thus, it is calculated:

$$
[\text { Ybus }]_{p u}=\left[\begin{array}{cccccc}
Y_{t} & 0 & 0 & \frac{-Y_{t}}{\sqrt{3}} & \frac{Y_{t}}{\sqrt{3}} & 0 \\
0 & Y_{t} & 0 & 0 & \frac{-Y_{t}}{\sqrt{3}} & \frac{Y_{t}}{\sqrt{3}} \\
0 & 0 & Y_{t} & \frac{Y_{t}}{\sqrt{3}} & 0 & \frac{-Y_{t}}{\sqrt{3}} \\
\frac{-Y_{t}}{\sqrt{3}} & 0 & \frac{Y_{t}}{\sqrt{3}} & \frac{2 Y_{t}}{3} & \frac{-Y_{t}}{3} & \frac{-Y_{t}}{3} \\
\frac{Y_{t}}{\sqrt{3}} & \frac{-Y_{t}}{\sqrt{3}} & 0 & \frac{-Y_{t}}{3} & \frac{2 Y_{t}}{3} & \frac{-Y_{t}}{3} \\
0 & \frac{Y_{t}}{\sqrt{3}} & \frac{-Y_{t}}{\sqrt{3}} & \frac{-Y_{t}}{3} & \frac{-Y_{t}}{3} & \frac{2 Y_{t}}{3}
\end{array}\right]
$$

\subsubsection{Isolated-neutral Yd1 Transformer}

In this case there exist 7 nodes: $a, b$ and $c$ at the primary side; $A, B$ and $C$ at the secondary side and the floating point $n$ (common point of wye connection).

Then, considering $V_{1}=V_{a}-V_{n}, V_{3}=V_{b}-V_{n}$ and $V_{5}=V_{c}-V_{n}$, a 7 by 7 admittance matrix in p.u. can be computed by using equation (19). Finally, using Kron's reduction for eliminating the floating point $n$, the following matrix is obtained:

$[\text { Ybus }]_{p u}=\left[\begin{array}{cccccc}\frac{2 Y_{t}}{3} & \frac{-Y_{t}}{3} & \frac{-Y_{t}}{3} & \frac{-Y_{t}}{\sqrt{3}} & \frac{Y_{t}}{\sqrt{3}} & 0 \\ \frac{-Y_{t}}{3} & \frac{2 Y_{t}}{3} & \frac{-Y_{t}}{3} & 0 & -\frac{Y_{t}}{\sqrt{3}} & \frac{Y_{t}}{\sqrt{3}} \\ \frac{-Y_{t}}{3} & \frac{-Y_{t}}{3} & \frac{2 Y_{t}}{3} & \frac{Y_{t}}{\sqrt{3}} & 0 & -\frac{Y_{t}}{\sqrt{3}} \\ -\frac{Y_{t}}{\sqrt{3}} & 0 & \frac{Y_{t}}{\sqrt{3}} & \frac{2 Y_{t}}{3} & \frac{-Y_{t}}{3} & \frac{-Y_{t}}{3} \\ \frac{Y_{t}}{\sqrt{3}} & -\frac{Y_{t}}{\sqrt{3}} & 0 & \frac{-Y_{t}}{3} & \frac{2 Y_{t}}{3} & \frac{-Y_{t}}{3} \\ 0 & \frac{Y_{t}}{\sqrt{3}} & -\frac{Y_{t}}{\sqrt{3}} & \frac{-Y_{t}}{3} & \frac{-Y_{t}}{3} & \frac{2 Y_{t}}{3}\end{array}\right]$

\subsection{Connections Summary}

\subsubsection{In Siemens}

The connections summary based on the four submatrices of $[Y$ bus $]$, i.e. $\left[Y_{p p}\right],\left[Y_{p s}\right],\left[Y_{s p}\right]$ and $\left[Y_{s s}\right]$, is shown in table 4. This is actually an extension of a table presented in
Table 4: Connections Summary for analysis in Siemens.

\begin{tabular}{|c|c|c|c|c|c|c|}
\hline Connection & {$\left[Y_{p p}\right]$} & {$\left[Y_{p p}\right]$} & {$\left[Y_{p p}\right]$} & {$\left[Y_{p p}\right]$} & $\begin{array}{c}\text { Angular } \\
\text { Disp. }\end{array}$ & $\begin{array}{c}\text { Clock } \\
\text { Hour }\end{array}$ \\
\hline$Y g-Y g$ & {$\left[Y_{I}\right]$} & {$\left[Y_{I}\right] \cdot a^{2}$} & $-\left[Y_{I}\right] \cdot a$ & $-\left[Y_{I}\right] \cdot a$ & $0^{o}$ & 0 \\
\hline$Y g-Y$ & {$\left[Y_{I I}\right]$} & {$\left[Y_{I I}\right] \cdot a^{2}$} & $-\left[Y_{I I}\right] \cdot a$ & $-\left[Y_{I I}\right] \cdot a$ & $0^{o}$ & 0 \\
\hline$Y g-D$ & {$\left[Y_{I}\right]$} & $3 \cdot\left[Y_{I I}\right] \cdot a^{2}$ & $\sqrt{3} \cdot\left[Y_{I I I}\right] \cdot a$ & $\sqrt{3} \cdot\left[Y_{I I I}\right]^{t} \cdot a$ & $30^{\circ}$ & 1 \\
\hline$Y-Y g$ & {$\left[Y_{I I}\right]$} & {$\left[Y_{I I}\right] \cdot a^{2}$} & $-\left[Y_{I I}\right] \cdot a$ & $-\left[Y_{I I}\right] \cdot a$ & $0^{o}$ & 0 \\
\hline$Y-Y$ & {$\left[Y_{I I}\right]$} & {$\left[Y_{I I}\right] \cdot a^{2}$} & $-\left[Y_{I I}\right] \cdot a$ & $-\left[Y_{I I}\right] \cdot a$ & $0^{o}$ & 0 \\
\hline$Y-D$ & {$\left[Y_{I I}\right]$} & $3 \cdot\left[Y_{I I}\right] \cdot a^{2}$ & $\sqrt{3} \cdot\left[Y_{I I I}\right] \cdot a$ & $\sqrt{3} \cdot\left[Y_{I I I}\right]^{t} \cdot a$ & $30^{\circ}$ & 1 \\
\hline$D-Y g$ & $3 \cdot\left[Y_{I I}\right]$ & {$\left[Y_{I}\right] \cdot a^{2}$} & $\sqrt{3} \cdot\left[Y_{I I I}\right]^{t} \cdot a$ & $\sqrt{3} \cdot\left[Y_{I I I}\right] \cdot a$ & $330^{\circ}$ & 11 \\
\hline$D-Y$ & $3 \cdot\left[Y_{I I}\right]$ & {$\left[Y_{I I}\right] \cdot a^{2}$} & $\sqrt{3} \cdot\left[Y_{I I I}\right]^{t} \cdot a$ & $\sqrt{3} \cdot\left[Y_{I I I}\right] \cdot a$ & $330^{\circ}$ & 11 \\
\hline$D-D$ & $3 \cdot\left[Y_{I I}\right]$ & $3 \cdot\left[Y_{I I}\right] \cdot a^{2}$ & $-3 \cdot\left[Y_{I I}\right] \cdot a$ & $-3 \cdot\left[Y_{I I}\right] \cdot a$ & $0^{\circ}$ & 0 \\
\hline
\end{tabular}

Chen et alli (1991) for per unit analysis and including the corresponding angular displacement.

Matrices presented in table 4, considering $Y_{t}$ in Siemens, are given by:

$$
\begin{gathered}
{\left[Y_{I}\right]=\left[\begin{array}{ccc}
Y_{t} & 0 & 0 \\
0 & Y_{t} & 0 \\
0 & 0 & Y_{t}
\end{array}\right],} \\
{\left[Y_{I I}\right]=\frac{1}{3}\left[\begin{array}{ccc}
2 Y_{t} & -Y_{t} & -Y_{t} \\
-Y_{t} & 2 Y_{t} & -Y_{t} \\
-Y_{t} & -Y_{t} & 2 Y_{t}
\end{array}\right]}
\end{gathered}
$$

and

$$
\left[Y_{I I I}\right]=\frac{1}{\sqrt{3}}\left[\begin{array}{ccc}
-Y_{t} & Y_{t} & 0 \\
0 & -Y_{t} & Y_{t} \\
Y_{t} & 0 & -Y_{t}
\end{array}\right]
$$

Notice that in general $\left[Y_{s p}\right]=\left[Y_{p s}\right]^{t}$. Given that $\left[Y_{I}\right]=\left[Y_{I}\right]^{t}$ and $\left[Y_{I I}\right]=\left[Y_{I I}\right]^{t}$ in some cases $\left[Y_{s p}\right]=\left[Y_{p s}\right]$.

\subsubsection{In Per Unit}

When an analysis in per unit is required, table 4 can be reduced, obtaining table 5 as presented in Chen et alli (1991).

Matrices $\left[Y_{I}\right],\left[Y_{I I}\right]$ and $\left[Y_{I I I}\right]$ are those of the previous approach (Siemens) while considering $Y_{t}$ in p.u.

Table 5 can be extended by including the angular displacement, as shown in table 4. It is important to remark that for those transformers fabricated under the ANSI 30 degrees, angular displacement for step-up Delta-wye or Wyedelta connections is $330^{\circ}$, whereas for step-down Delta-wye or Wye-delta connection is $30^{\circ}$. Consequently, step-down Delta-wye and step-up Wye-delta transformers that do not 
Table 5: Connections Summary, Chen et alli (1991).

\begin{tabular}{|c|c|c|c|c|}
\hline Connection & {$\left[Y_{p p}\right]$} & {$\left[Y_{p p}\right]$} & {$\left[Y_{p p}\right]$} & {$\left[Y_{p p}\right]$} \\
\hline$Y g-Y g$ & {$\left[Y_{I}\right]$} & {$\left[Y_{I}\right]$} & $-\left[Y_{I}\right]$ & $-\left[Y_{I}\right]$ \\
\hline$Y g-Y$ & {$\left[Y_{I I}\right]$} & {$\left[Y_{I I}\right]$} & $-\left[Y_{I I}\right]$ & $-\left[Y_{I I}\right]$ \\
\hline$Y g-D$ & {$\left[Y_{I}\right]$} & {$\left[Y_{I I}\right]$} & {$\left[Y_{I I I}\right]$} & {$\left[Y_{I I I}\right]^{t}$} \\
\hline$Y-Y g$ & {$\left[Y_{I I}\right]$} & {$\left[Y_{I I}\right]$} & $-\left[Y_{I I}\right]$ & $-\left[Y_{I I}\right]$ \\
\hline$Y-Y$ & {$\left[Y_{I I}\right]$} & {$\left[Y_{I I}\right]$} & $-\left[Y_{I I}\right]$ & $-\left[Y_{I I}\right]$ \\
\hline$Y-D$ & {$\left[Y_{I I}\right]$} & {$\left[Y_{I I}\right]$} & {$\left[Y_{I I I}\right]$} & {$\left[Y_{I I I}\right]^{t}$} \\
\hline$D-Y g$ & {$\left[Y_{I I}\right]$} & {$\left[Y_{I}\right]$} & {$\left[Y_{I I I}\right]^{t}$} & {$\left[Y_{I I I}\right]$} \\
\hline$D-Y$ & {$\left[Y_{I I}\right]$} & {$\left[Y_{I I}\right]$} & {$\left[Y_{I I I}\right]^{t}$} & {$\left[Y_{I I I}\right]$} \\
\hline$D-D$ & {$\left[Y_{I I}\right]$} & {$\left[Y_{I I}\right]$} & $-\left[Y_{I I}\right]$ & $-\left[Y_{I I}\right]$ \\
\hline
\end{tabular}

match the base angular displacement should be corrected as described thereafter in subsection 6.7.

\subsection{Ideal ordering for the primitive admittance and connection matrices}

For three-phase transformers the primitive admittance matrix $[Y p]$, as presented in equation (18), is ordered by grouping firstly the primary side parameters and then the secondary side. Thus, the new order of the rows and columns will be 1-3-5-2-4-6:

$$
\begin{aligned}
& {[Y p]=} \\
& \begin{array}{c}
1 \\
3 \\
5 \\
2 \\
4 \\
6
\end{array}\left[\begin{array}{cccccc}
1 & 3 & 5 & 2 & 4 & 6 \\
0 & 0 & 0 & -\mathrm{aYt} & 0 & 0 \\
0 & 0 & 0 & 0 & -\mathrm{aYt} & 0 \\
-\mathrm{aYt} & 0 & 0 & 0 & 0 & -\mathrm{aYt} \\
0 & -\mathrm{aYt} & 0 & 0 & \mathrm{a}^{2} \mathrm{Yt} & 0 \\
0 & 0 & -\mathrm{aYt} & 0 & 0 & \mathrm{a}^{2} \mathrm{Yt}
\end{array}\right] \\
& {[Y p]=\left[\begin{array}{ll}
{\left[Y_{p p}\right]_{\text {prim }}} & {\left[Y_{\text {ps }}\right]_{\text {prim }}} \\
{\left[Y_{s p}\right]_{\text {prim }}} & {\left[Y_{s s}\right]_{\text {prim }}}
\end{array}\right]}
\end{aligned}
$$

For any connection, it can be noticed that the four submatrices in equation (25) are diagonal:

$$
\begin{gathered}
{\left[Y_{p p}\right]_{\text {prim }}=Y t[I],} \\
{\left[Y_{p s}\right]_{\text {prim }}=-a Y t[I],} \\
{\left[Y_{s p}\right]_{\text {prim }}=-a Y t[I]}
\end{gathered}
$$

and

$$
\left[Y_{s s}\right]_{\text {prim }}=a^{2} Y t[I] .
$$

In addition, given the diagonal nature of $[Y p]$, it is verified that $\left[Y_{p s}\right]_{\text {prim }}=\left[Y_{s p}\right]_{\text {prim }}$.

Thus,

$$
[Y p]=\left[\begin{array}{cc}
Y t[I] & -a Y t[I] \\
-a Y t[I] & a^{2} Y t[I]
\end{array}\right]
$$

Analysis in per unit requires $a=1$ and $Y t$ in p.u., otherwise $a$ is the windings ratio and $Y t$ the admittance referred to the primary side (in Siemens).

This new re-ordering makes the connection matrix $[N]$ to present other particularities:

$$
[N]=\left[\begin{array}{cc}
{\left[N_{p p}\right]} & {[0]} \\
{[0]} & {\left[N_{s s}\right]}
\end{array}\right]
$$

Submatrices [0] are due to the absence of connections between primary and secondary sides in three-phase transformers. However, this would not be the case for autotransformers.

In order to compute [Ybus], equation (19) is solved by using equations (25) and (26):

$$
\begin{array}{r}
{[\text { Ybus }]=\left[\begin{array}{cc}
{\left[N_{p p}\right]^{t}} & {[0]} \\
{[0]} & {\left[N_{s s}\right]^{t}}
\end{array}\right] \cdot\left[\begin{array}{cc}
{\left[Y_{p p}\right]_{\text {prim }}} & {\left[Y_{\text {ps }}\right]_{\text {prim }}} \\
{\left[Y_{s p}\right]_{\text {prim }}} & {\left[Y_{\text {ss }}\right]_{\text {prim }}}
\end{array}\right]} \\
\cdot\left[\begin{array}{cc}
{\left[N_{p p}\right]} & {[0]} \\
{[0]} & {\left[N_{s s}\right]}
\end{array}\right]
\end{array}
$$

$$
[\text { Ybus }]=\left[\begin{array}{cc}
Y t\left[N_{p p}\right]^{t}\left[N_{p p}\right] & -a Y t\left[N_{p p}\right]^{t}\left[N_{s s}\right] \\
-a Y t\left[N_{s s}\right]^{t}\left[N_{p p}\right] & a^{2} Y t\left[N_{s s}\right]^{t}\left[N_{s s}\right]
\end{array}\right]
$$

Equation (27) is valid in Siemens. Per unit analysis should consider $a=1, Y t$ in p.u., and some submatrices should be modified according to subsection 6.2.

Given that, from matrices theory, $(A \cdot B)^{t}=B^{t} \cdot A^{t}$ then:

$$
\left(\left[N_{p p}\right]^{t}\left[N_{s s}\right]\right)^{t}=\left[N_{s s}\right]^{t}\left(\left[N_{p p}\right]^{t}\right)^{t}=\left[N_{s s}\right]^{t}\left[N_{p p}\right] .
$$

Consequently, it is observed that for $[Y b u s]$ always $\left[Y_{s p}\right]=$ $\left[Y_{p s}\right]^{t}$. 
Table 6: Two winding three-phase transformer vector groups.

\begin{tabular}{|c|c|}
\hline Vector Group & Clock Hours \\
\hline I & $0-4-8$ \\
\hline II & $2-6-10$ \\
\hline III & $1-5-9$ \\
\hline IV & $3-7-11$ \\
\hline
\end{tabular}

\subsection{Incidence of the Angular Displacement}

There are two ways for including the angular displacement in the transformer modeling:

a. A connection matrix $[N]$ is constructed taking into account the particular angular displacement under study. Then (19) is solved for either p.u. or siemens.

b. First, a connection summary is used (table 4 or table 5). Then, by considering the corresponding basic clock hour (angular displacement) and the appropriate methodology, any other clock hour can be achieved.

When equation (19) is used in order to compute [Ybus] for each of the 26 IEC connections (excluding 10 zig-zag connections) it is observed that submatrices $\left[Y_{p p}\right]$ and $\left[Y_{s s}\right]$ do not suffer any modification due to changes in angular displacement. Consequently, given that $\left[Y_{s p}\right]=\left[Y_{p s}\right]^{t}$ it is necessary to solely focus on analyzing $\left[Y_{p s}\right]$.

Starting from the basic clock hour, in order to "move" a given clock hour to the next one within the same vector group (see table 6), rows of the submatrix $\left[Y_{p s}\right]$ should be "rotated" in such a way that the first row becomes the second, the second becomes the third and the third becomes the first. If the desired clock hour was not achieved yet, the process is repeated considering the new matrix (obtained in the previous step) as the basic one.

If a vector group change is required, firstly the $\left[Y_{p s}\right]$ should be obtained considering a connection 6 clock hours above the basic clock hour, which is the negative of it. In this way:

- For Yy or Dd connection, whose basic clock hour is 0 (table 4), the corresponding 6-clock-hours-above submatrix is: $\left[Y_{p s}\right]_{6 o^{\prime} \text { clock }}=-\left[Y_{p s}\right]_{0 o^{\prime} \text { clock }}$.
- For Yd connection, whose basic clock hour is 1: $\left[Y_{p s}\right]_{7 o^{\prime} \text { clock }}=-\left[Y_{p s}\right]_{1 o^{\prime} \text { clock }}$.

- For Dy connection, whose basic clock hour is 11: $\left[Y_{p s}\right]_{5 o^{\prime} \text { clock }}=-\left[Y_{p s}\right]_{11 o^{\prime} \text { clock }}$.

If after the vector group change another clock hour (within the same vector group) is required, the procedure explained previously should be performed.

\subsection{Incidence of taps in the three-phase transformer modeling}

When considering off-nominal taps, matrix $[Y b u s]$ from equation (20) is modified into:

$$
[\text { Ybus }]=\left[\begin{array}{ll}
{\left[Y_{p p}\right] / \alpha^{2}} & {\left[Y_{p s}\right] / \alpha \beta} \\
{\left[Y_{s p}\right] / \alpha \beta} & {\left[Y_{s s}\right] / \beta^{2}}
\end{array}\right]
$$

This can be demonstrated by extending the procedure presented in subsection 4.2, for single-phase transformer, to the three-phase transformer analysis.

Thus, the corresponding primitive admittance matrix is:

$$
[Y p]=\left[\begin{array}{cccccc}
\frac{Y t}{\alpha^{2}} & \frac{-a Y t}{\alpha \beta} & 0 & 0 & 0 & 0 \\
\frac{-a Y t}{\alpha \beta} & \frac{a^{2} Y t}{\beta^{2}} & 0 & 0 & 0 & 0 \\
0 & 0 & \frac{Y t}{\alpha^{2}} & \frac{-a Y t}{\alpha \beta} & 0 & 0 \\
0 & 0 & \frac{-a Y t}{\alpha \beta} & \frac{a^{2} Y t}{\beta^{2}} & 0 & 0 \\
0 & 0 & 0 & 0 & \frac{Y t}{\alpha^{2}} & \frac{-a Y t}{\alpha \beta} \\
0 & 0 & 0 & 0 & \frac{-a Y t}{\alpha \beta} & \frac{a^{2} Y t}{\beta^{2}}
\end{array}\right]
$$

Re-ordering rows and columns based on primary and secondary sides, the new primitive admittance matrix is:

$[Y p]=\left[\begin{array}{cccccc}\frac{Y t}{\alpha^{2}} & 0 & 0 & \frac{-a Y t}{\alpha \beta} & 0 & 0 \\ 0 & \frac{Y t}{\alpha^{2}} & 0 & 0 & \frac{-a Y t}{\alpha \beta} & 0 \\ 0 & 0 & \frac{Y t}{\alpha^{2}} & 0 & 0 & \frac{-a Y t}{\alpha \beta} \\ \frac{-a Y t}{\alpha \beta} & 0 & 0 & \frac{a^{2} Y t}{\beta^{2}} & 0 & 0 \\ 0 & \frac{-a Y t}{\alpha \beta} & 0 & 0 & \frac{a^{2} Y t}{\beta^{2}} & 0 \\ 0 & 0 & \frac{-a Y t}{\alpha \beta} & 0 & 0 & \frac{a^{2} Y t}{\beta^{2}}\end{array}\right]$.

Given that the four primitive submatrices $\left[Y_{p p}\right]_{\text {prim }}$, $\left[Y_{p s}\right]_{\text {prim }},\left[Y_{s p}\right]_{\text {prim }}$ and $\left[Y_{s s}\right]_{\text {prim }}$ are diagonal:

$$
[Y p]=\left[\begin{array}{cc}
Y t / \alpha^{2}[I] & -a Y t / \alpha \beta[I] \\
-a Y t / \alpha \beta[I] & a^{2} Y t / \beta^{2}[I]
\end{array}\right] .
$$


By using equation (26) and solving equation (19), it is obtained:

$[Y$ bus $]=\left[\begin{array}{cc}Y t / \alpha^{2}\left[N_{p p}\right]^{t}\left[N_{p p}\right] & -a Y t / \alpha \beta\left[N_{p p}\right]^{t}\left[N_{s s}\right] \\ -a Y t / \alpha \beta\left[N_{s s}\right]^{t}\left[N_{p p}\right] & a^{2} Y t / \beta^{2}\left[N_{s s}\right]^{t}\left[N_{s s}\right]\end{array}\right] \cdot$ Dugan

Thus, submatrix $\left\lfloor Y_{p p}\right\rfloor$ is divided by $\alpha^{2}$, submatrices $\left\lfloor Y_{p s}\right\rfloor$ and $\left\lfloor Y_{s p}\right\rfloor$ are divided by $\alpha \beta$ and submatrix $\left[Y_{s s}\right]$ is divided by $\beta^{2}$. This is valid for all clock hours, both in per unit or Siemens.

\section{CONCLUSIONS}

A methodology that allows, with minimal data (one shortcircuit test), modeling three-phase distribution and power transformers based on a single-phase transformers bank was presented. Proposed models provide detailed information required for implementing most distribution transformer connections. In addition, a methodology to include angular displacement (basic tool for generalizing transformer models to other different clock hours or even vector groups) and tapping incidence, commonly not addressed in the specialized literature (Wang et alli, 2004; Xiao et alli, 2006), was presented. Both approaches, actual values and per unit, were also addressed aimed at specifying the corresponding differences.

\section{REFERENCES}

Baran, M. E. and E. A. Staton. (1997). Distribution transformer models for branch current based feeder analysis. IEEE Transactions on Power Systems, Vol. 12, No. 2, pp. 698-703.

Chen, T.-H.; Chen, M.-S.; Inoue, T.; Kotas, P. and Chebli, E. A. (1991). Three-phase cogenerator and transformer models for distribution system analysis. IEEE Transactions on Power Delivery, Vol. 6, No. 4, pp. 1671-1681.

Chen, T.-H. and Chang, J.-D. (1992). Open wye-open delta and open delta-open delta transformer modelsfor rigorous distribution system analysis. IEE Proceedings -Generation, Transmission and Distribution, Vol. 139, No. 3, pp. 227-234.

Chen, T.-H.; Chang, J.-D. and Chang, Y.-L. (1996). Models of grounded mid-tap open-wye and opendelta connectedtransformers for rigorous analysis of a distribution system. IEE Proceedings -Generation, Transmission and Distribution, Vol. 143, No. 1, pp. 1350-2360, Jan. 1996.
Chen, T.-H. and Chang, Y.-L. (1996). Integrated models of distribution transformers and their loads forthree-phase power flow analyses. IEEE Transactions on Power Delivery, Vol. 11, No. 1, pp. 507-513.

Dugan, R. C. (2003). A perspective on transformer modeling for distribution system analysis. IEEE Power Engineering Society General Meeting, pp. 114-119.

Gorman, M. J. and Grainger, J. J. (1992). Transformer Modelling for Distribution System Studies, Part I: Linear Modelling Basics. Part II: Addition of Models to Ybus and Zbus. IEEE Transactions on Power Delivery, Vol. 7, No. 2, pp. 575-580.

Kersting, W. H.; Phillips, W. H. and Carr, W. (1999). A new approach to modeling three-phase transformer connections. IEEE Transactions on Industry Applications, Vol. 35, No. 1, pp. 169-175.

Kersting, W. H. (2002). Distribution System Modeling and Analysis. CRC Press LLC, Florida.

Wang, Z.; Chen, F.; and Li, J. (2004). Implementing transformer nodal admittance matrices into backward/forward sweep-based power flow analysis for unbalanced radial distribution systems. IEEE Transactions on Power Systems, Vol. 19, No. 4, pp. 1831-1836.

Xiao, P.; Yu, D. C.; and Yan, W. (2006). A unified threephase transformer model for distribution load flow calculations. IEEE Transactions on Power Systems, Vol. 21, No. 1, pp. 153-159. 